\title{
Distinguishing the Sources
}

\begin{abstract}
Our discussion of history has covered many of the observations that have led to the ideas of acceleration by shock waves or by magnetic reconnection in gradual and impulsive solar energetic particle (SEP) events, respectively. We now present other compelling observations, including onset timing, SEP-shock correlations, injection time profiles, high-energy spectral knees, e/p ratios, and intensity dropouts caused by a compact source, that have helped clarify these acceleration mechanisms and sources. However, some of the newest evidence now comes from source-plasma temperatures. In this and the next two chapters, we will find that impulsive events come from solar active regions at $\approx 3 \mathrm{MK}$, controlling ionization states $Q$, hence $A / Q$, and, in most gradual events, shocks accelerate ambient coronal material from $\leq 1.6 \mathrm{MK}$. When SEPs are trapped on closed loops they supply the energy for flares. In addition to helping to define their own origin, SEPs also probe the structure of the interplanetary magnetic field.
\end{abstract}

The history in Chap. 2 suggested how the flow of observations and ideas eventually led to credible evidence of two sites of SEP acceleration and the related physical mechanisms. While some observations have been described, some of the clearest evidence of origin has not yet been presented. In this chapter we continue the story of particle origin, showing where and when SEPs are accelerated, and measurements that allow us to compare impulsive and gradual events. There are many different lines of evidence that fit together to determine the most probable origins, and that evidence continues to grow. 


\subsection{SEP Onset Times}

Even in relatively intense SEP events, it is likely that the earliest detectible particles at each energy will be those that were originally focused in the diverging magnetic field in the inner heliosphere and have scattered least, simply traversing along the magnetic field line from the source with an average pitch-angle cosine, $\langle\mu\rangle \approx 1$, so that they arrive first. An example of the observed arrival times of particles of different energies is shown in Fig. 3.1. The rise of the intensities is clear and sharp and intensities rise by two or three orders of magnitude. If $1 \%$ or more of the ions in each energy interval have traveled with $\langle\mu\rangle \approx 1$, we will be able to determine the scatter-free onset time with reasonable accuracy. The accuracy of this scatter-free approximation has been well studied and will be discussed below. As discussed in Sect. 2.3.4, impulsive events are all almost entirely scatter-free. The particle transit time $t=L / v$ where $L$ is the path length along the field line and $v$ is the particle velocity. By fitting the measurements we can determine both the path length and the time that the particles left the Sun, the so-called solar particle release (SPR) time.

Note that the SPR time is the release time at the Sun; to compare with photon observation times at Earth one should add 8.3 min to the SPR time. The path length of $1.11 \pm 0.02 \mathrm{AU}$ allows for some curvature of the Parker spiral, typically 1.1-1.2 AU. For large gradual events, including the ground level events (GLEs), generally the SPR times occur quite late in the event. Timing in impulsive (left) and gradual (right) events is compared in Fig. 3.2.

For the impulsive SEP events in Fig. 3.2, the SPR times fall rather precisely on the hard-X-ray peak times (there are no measurable $\gamma$-rays in these events). For the
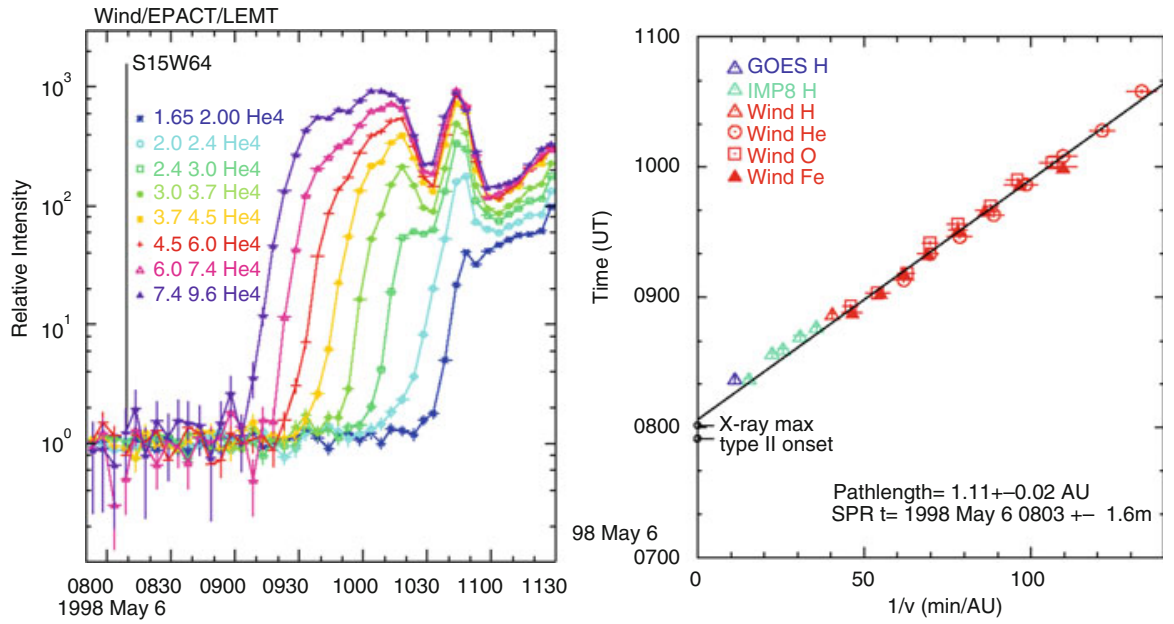

Fig. 3.1 The left panel shows the arrival of ${ }^{4} \mathrm{He}$ ions of the indicated $\mathrm{MeV} \mathrm{amu}{ }^{-1}$ intervals at the Wind spacecraft near Earth. The right panel shows the onset time of these and other intervals vs. $v^{-1}$. For the fitted line, the slope is the pathlength and the intercept is the solar particle release (SPR) time at the Sun for this large gradual GLE event (Reames 2009a, (C) AAS) 

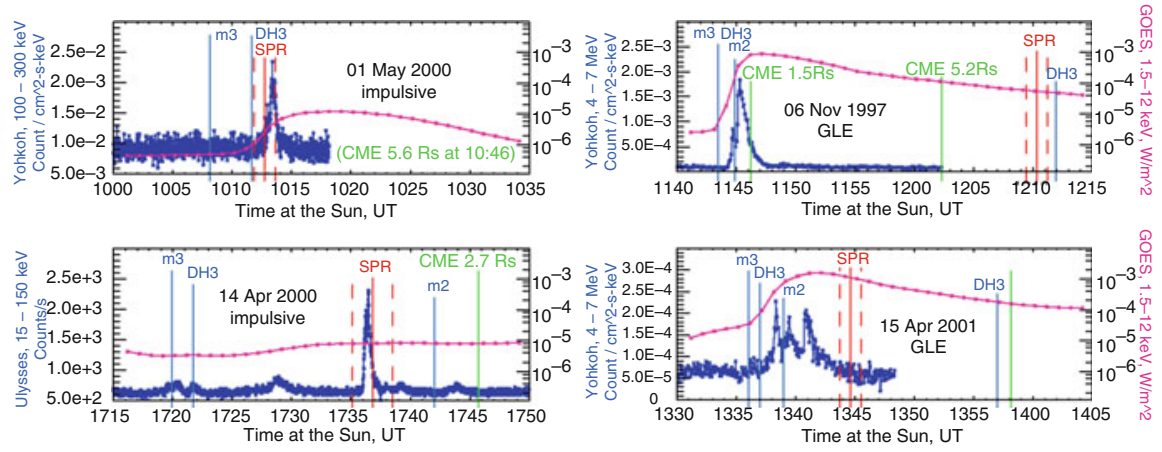

Fig. 3.2 A comparison is shown of timing in two impulsive (left) and two gradual (right) SEP events. Solar particle release (SPR) times of the particles (red with dashed errors) are compared with hard X-ray (dark blue, left), $\gamma$-ray (dark blue, right) and GOES soft X-ray (violet) time profiles. Onset times of metric (m) and decametric-hectometric (DH; 1-14 MHz) type II and III radio bursts (light blue) and CME locations (green) are shown (adapted from Tylka et al. 2003)
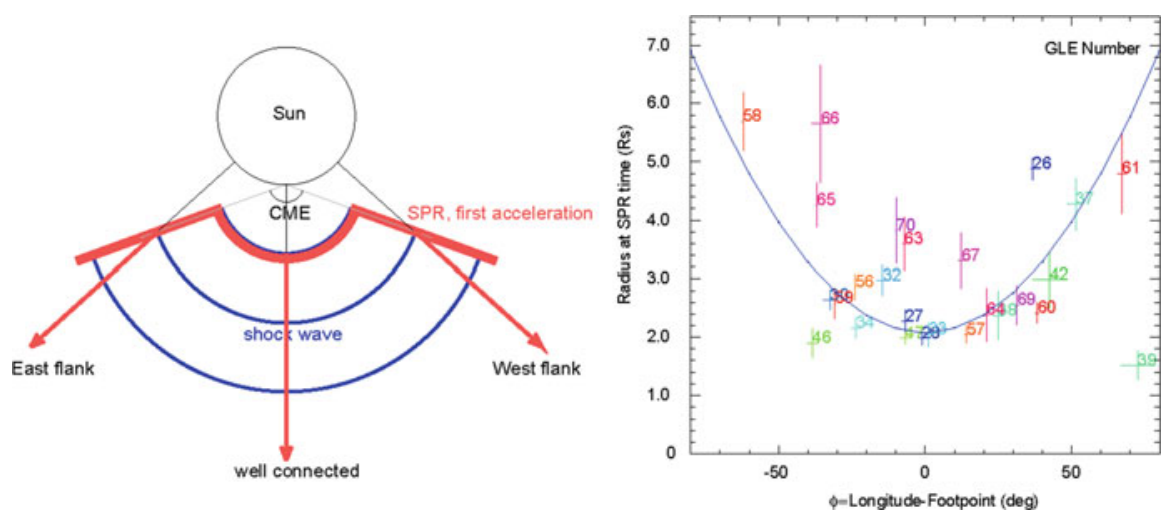

Fig. 3.3 The right panel shows the height of the CME at SPR time vs. longitude for numbered GLEs. The cartoon on the left shows the CME and SPR location widening on the flanks (Reames 2009a, b (C) AAS). The height distribution is fit to a symmetric parabola for comparison; actually a height of $2-3 R_{S}$ is fairly constant over $\sim 70^{\circ}$. This could be the width of the source shock surface above closed loops that was once incorrectly called the "fast propagation region". SPR may differ on each local SEP field line, while type II onset occurs near the source longitude

GLEs, the SPR times often fall well after the $\gamma$-rays are over (by up to $30 \mathrm{~min}$ ), but always after the metric type II onset indicates the formation of a shock wave. Rouillard et al. (2016) relate the SPR delay to the time required for the shock to become supercritical, i.e. Mach $>3$.

It is interesting to plot the height of the CME leading edge at SPR time as a function of longitude of the observer relative to that of the CME source as shown in the right panel of Fig. 3.3 for the GLEs. For a multi-spacecraft study of a single event see Reames and Lal (2010). 
Clearly a correct estimate of the SPR time depends upon the intensities being sufficiently high that a small number of un-scattered ions are detectible. Gopalswamy et al. (2012) have simply assumed a path length of $1.2 \mathrm{AU}$ in order to avoid the velocity-dispersion analysis. However, Rouillard et al. (2012, see Appendix) have calculated that the error in the SPR time from scattering should be less that 1-2 min., comparable with errors from the 5 min-averaged data used. If scattering delayed low energies more, the apparent SPR would be too early. Note also that the impulsive events on the left in Fig. 3.2 show no evidence of onset errors. Tan et al. (2013) have found that the SPR times and path lengths of the non-relativistic electrons agree with those of the ions, and Rouillard et al. (2012) have also shown lateral spreading of the shock wave as imaged by the coronagraphs.

High-energy $(\mathrm{GeV})$ protons are often strongly beamed along the interplanetary magnetic-field $\boldsymbol{B}$, so a particular neutron monitor on Earth sees a peak when its asymptotic look direction is aligned with $\boldsymbol{B}$. As $\boldsymbol{B}$ varies, neutron monitors often see spiky increases or multiple peaks and valleys of intensity.

Surely there are a few GLEs where the SPR timing alone would permit some kind of (unspecified) acceleration at the time of the associated flare. However, these events may just have faster CMEs or a faster decrease in $V_{\mathrm{A}}$ with radius that would permit earlier ion acceleration by the shock, or earlier arrival of the shock above closed magnetic loops. If the GLEs with late SPR times are clearly shock accelerated, why would we seek a new mechanism for those events with earlier SPR times which have equally strong shocks? Shock acceleration is able to account for SEP acceleration in all gradual events, including GLEs, especially in GLEs. No other mechanism is required, no other seems capable.

\subsection{Realistic Shock-SEP Timing and Correlations}

With recent measurements on the STEREO spacecraft, it has been possible to construct three-dimensional distributions of CMEs and shocks and compare them with SEPs, i.e. to compare the SEPs and the shock along the same single field line (Rouillard et al. 2011, 2012, 2016). Figure 3.4 shows aspects of this comparison.

The left-hand simulation in Fig. 3.4 reconstructs the way the CME and shock spread. The actual SPR time depends upon the time an active shock actually strikes the (dashed) field line to an observer. It would be a great improvement on the comparison in Fig. 3.3 if we could see the local shock as we can here. Some images of the shock are shown in the upper right panels of Fig. 3.4.

The lower-right panels in Fig. 3.4 show correlations of peak proton intensities at 2 and $20 \mathrm{MeV}$ with CME speed from the earlier study by Kahler (2001) shown in Fig. 2.12. However, no single speed exists for any CME or shock, and there is no single peak proton intensity, since both vary strongly with longitude. The red points in the lower right panel of Fig. 3.4 compare intensity and CME speed on single magnetic flux tubes, apparently improving the correlation. Kouloumvakos et al. (2019) have extended the CME-SEP correlations by modeling the 3D geometry of 

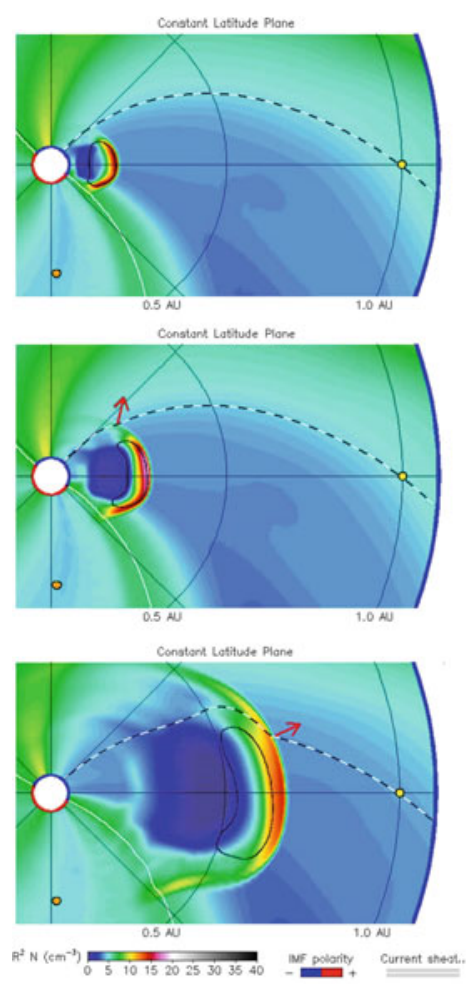
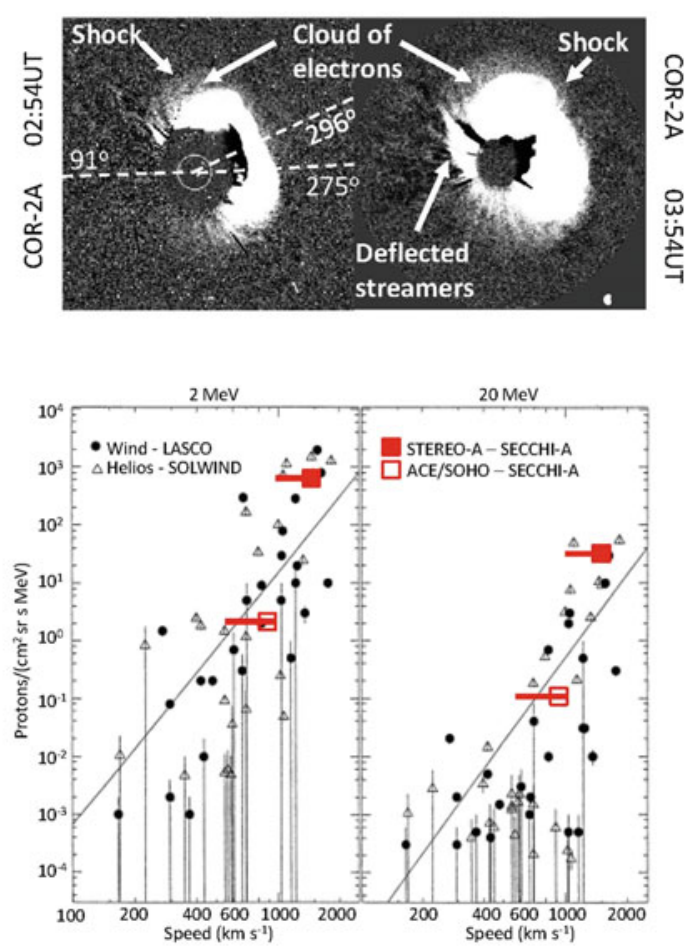

Fig. 3.4 Left-hand panels show a STEREO simulation of the evolution and lateral spread of the CME and shock. Upper-right panels show actual images of the shock. Lower right panels show possible improvement in the intensity-CME-speed correlation (red points) when observed on single field lines at two different longitudes in an event (Rouillard et al. 2011, 2012 (C) AAS)

shock waves using the three coronagraph images from SOHO/LASCO and STEREO A and B, as noted in Sect. 2.10.

More recently, Gopalswamy et al. (2013) studied the first GLE of Solar Cycle 24, GLE 71 on 17 May 2012, together with 6 other large, well-connected events with fast CMEs. The evolution of two of the CMEs is compared in Fig. 3.5.

In the GLE, the shock formation height (type II radio burst onset) is at $1.38 \mathrm{R}_{\mathrm{S}}$ and the observed CME height at the time of particle release was directly measured as $2.32 \mathrm{R}_{\mathrm{S}}$. This is consistent with the findings from extrapolations of GLEs in Cycle 23. The authors concluded that the event of May 2012 was a GLE simply because it was better connected to Earth than the other large SEP events with similar or even faster CMEs. Note that the SPR time is the SEP onset on the local field-line longitude of the SEP observer, while the type II onset is at a longitude nearest the source; hence, the type II onsets do not show the quasi-parabolic behavior of the SPR height in Fig. 3.2.

Thakur et al. (2016, see also Tylka and Dietrich 2009) compared the $>700 \mathrm{MeV}$ proton channel on GOES as an alternate indicator of GLEs. They found two events 


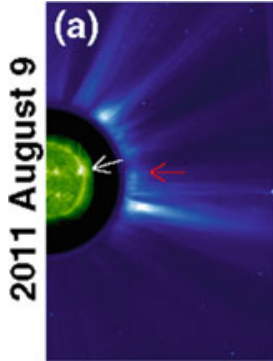

08:12

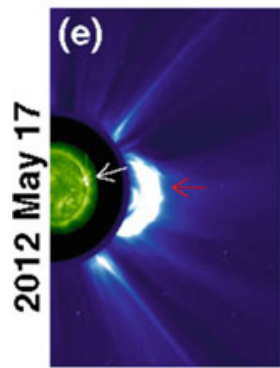

01:48

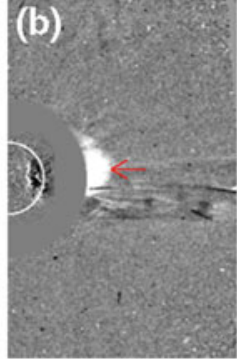

08:12

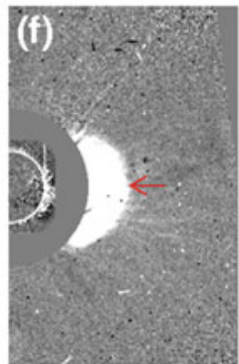

01:48

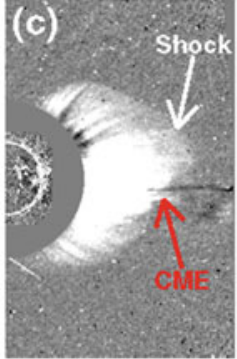

$08: 24$

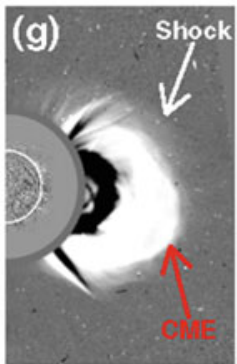

02:00

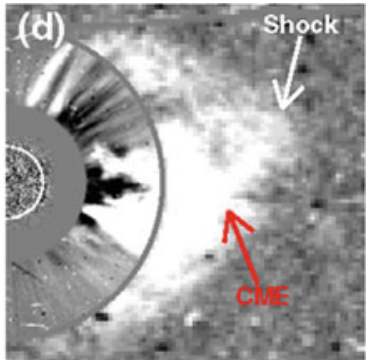

08:42

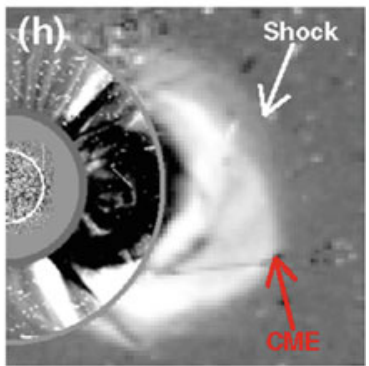

02:18

Fig. 3.5 The time evolution of two CMEs, 9 August 2011 and the GLE on 17 May 2012, are shown from their first appearance on SOHO/LASCO. In panels (a) and (e), the SDO/AIA solar image at $195 \AA$ shows the solar sources while the remaining difference images show evolution of the CMEs and shocks. Red arrows point to the CME nose. The shock remains closer to the CME in (h) than in (d), indicating a stronger shock (Gopalswamy et al. 2013 (C) AAS)

that differed, one GLE with no increase at $>700 \mathrm{MeV}$ and one $>700-\mathrm{MeV}$ increase that was not a GLE. They ascribed the difference to the level of the background. They also found that GLEs were generally observed when the shocks form at $1.2-1.93 \mathrm{R}_{\mathrm{S}}$ and when solar particle release (SPR) occurs between $2-6 \mathrm{R}_{\mathrm{S}}$. Note that the electron acceleration that produces the type II burst could occur while the shock is still propagating within closed magnetic loops, but SPR time must occur where the shock is on open field lines, and at a local longitude.

Cliver et al. (2004) found a strong ( 90\%) association of decametric-hectometric (DH; 1-14 MHz) type II radio emission produced at $\sim 3 \mathrm{R}_{\mathrm{S}}$ by SEP events with $20 \mathrm{MeV}$ protons (see Fig. 2.1). The correlation was only $25 \%$ for lower-altitude metric type II's without $\mathrm{DH}$, suggesting that shock acceleration is strongest at or above $\sim 3 \mathrm{R}_{\mathrm{S}}$.

\subsection{Injection Profiles}

Relating to SEP increases early in events, Kahler (1994) plotted the intensity of SEPs, not as a function of time, but as a function of the height of the CME, using the height-time plot for the CME, as shown in Fig. 3.6. Not only are the protons injected 
Fig. 3.6 Injection profiles of high-energy protons are shown as a function of CME height for three GLEs in 1989: August 16, September 29, and October 24. The CME speeds for these events are 1377 , 1828 , and $1453 \mathrm{~km} \mathrm{~s}^{-1}$, respectively (Kahler 1994, (C) AAS)

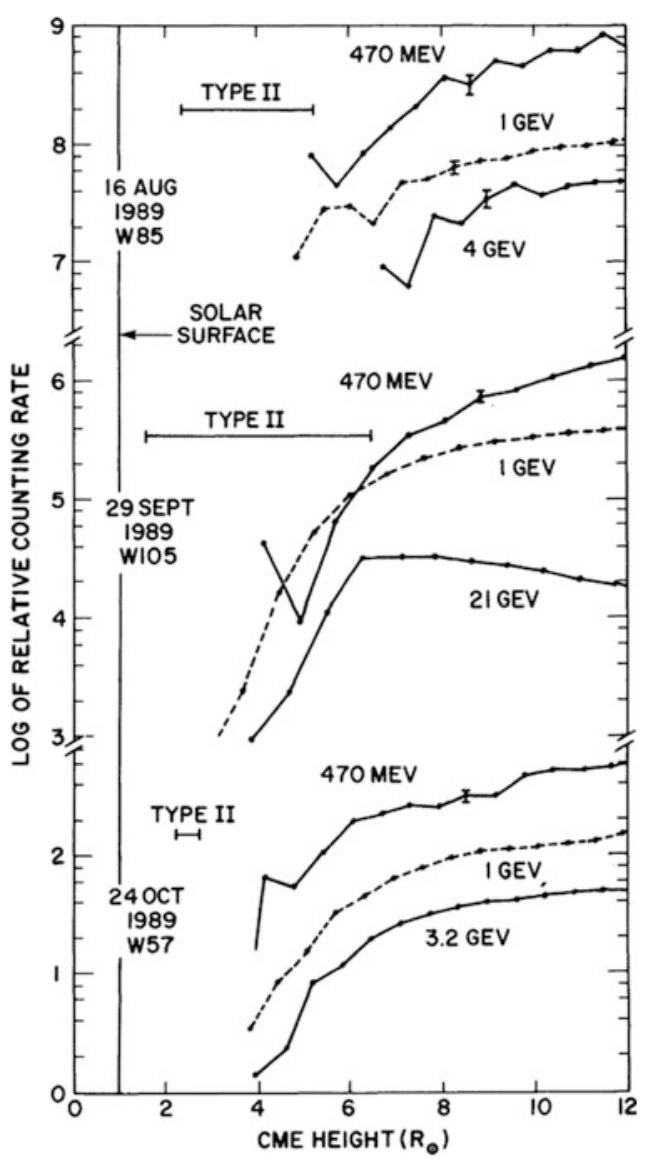

late, but their intensities continue to rise until $R>6 \mathrm{R}_{\mathrm{S}}$, even at $21 \mathrm{GeV}$. A final peak in the Alfvén speed vs. height occurs at $\sim 4 \mathrm{R}_{\mathrm{S}}$ and $V_{\mathrm{A}}$ has probably declined to about $600 \mathrm{~km} \mathrm{~s}^{-1}$ at $6 \mathrm{R}_{\mathrm{S}}$ (Mann et al. 2003; see also Sect. 1.4). The Alfvén-Mach number of the shock, $V_{\mathrm{S}} / V_{\mathrm{A}}$ remains at $\sim 2$ or greater above $\sim 1.2 \mathrm{R}_{\mathrm{S}}$ for these shock waves.

\subsection{High-Energy Spectra and Spectral Knees}

Are GLEs fundamentally different from other gradual SEP events? Is it likely that there is some new source of particles that can only be seen at energies above $\sim 0.5 \mathrm{GeV}$ ? Much of the evidence connecting gradual SEPs to shock acceleration, especially element abundances and source-plasma temperatures, comes from energies below $100 \mathrm{MeV}$. Do the high-energy spectra come from the same source? Some spectra are shown in Fig. 3.7.

Mewaldt et al. (2012) studied spectra and element abundances of 16 GLEs. They found that the empirical double power-law spectral forms give a better fit than the 

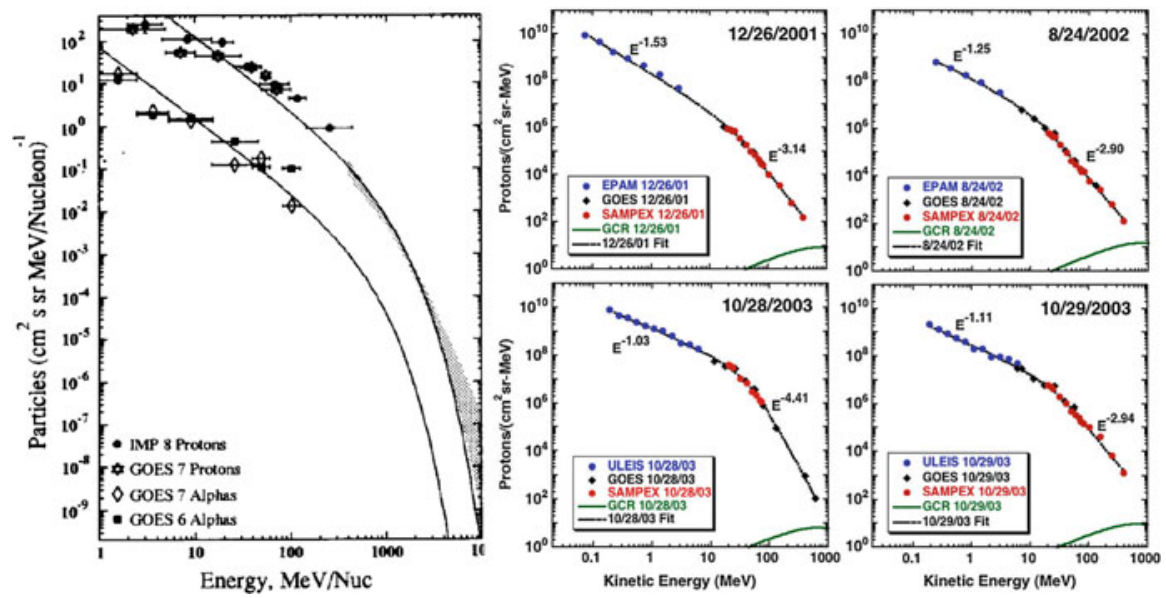

Fig. 3.7 The left panel shows H and He in the large GLE of September 29, 1989 (Lovell et al. 1998). The shaded region is the spectrum deduced from neutron monitors and the spectra are fit to the shock-spectral shape of Ellison and Ramaty (1985). The four right-hand panels show GLE fluence spectra that are typical of the 16 GLE spectra assembled by Mewaldt et al. (2012 (C) Springer) fit to double power-law spectra

power-law-times-exponential spectrum of Ellison and Ramaty (1985, see also Lee 2005 ) that models escape of high-energy particles from the shock. In any case, none of the 16 GLEs showed evidence of high-energy spectral hardening that might suggest the existence of a new source that could dominate higher energies.

Tylka and Dietrich (2009) have used the geomagnetic cutoff rigidities at neutronmonitor stations to develop integral rigidity spectra, using data from the world-wide neutron monitor network for 53 GLEs. The proton spectra are fit to double power laws in rigidity, decreasing with a power above $1 \mathrm{GV}(430 \mathrm{MeV})$ in the range of 5-7 in $70 \%$ of the GLEs (see Sect. 6.1). None show hardening.

\subsection{Intensity Dropouts and Compact Sources}

When Mazur et al. (2000) plotted the energy of individual ions as a function of their arrival time, as seen in Fig. 3.8, they found that the pattern of velocity dispersion that we described in Fig. 3.1 was sharply interrupted for time intervals when the spacecraft was simply not magnetically connected to the particle source. This was seen for impulsive SEP events and would be expected if magnetic flux tubes that were connected to a compact source were interspersed with others that were not, as confirmed by theory (Giacalone et al. 2000). Gaps were not often seen in gradual events where a spatially extensive shock wave would be expected to populate all field lines with SEPs. 

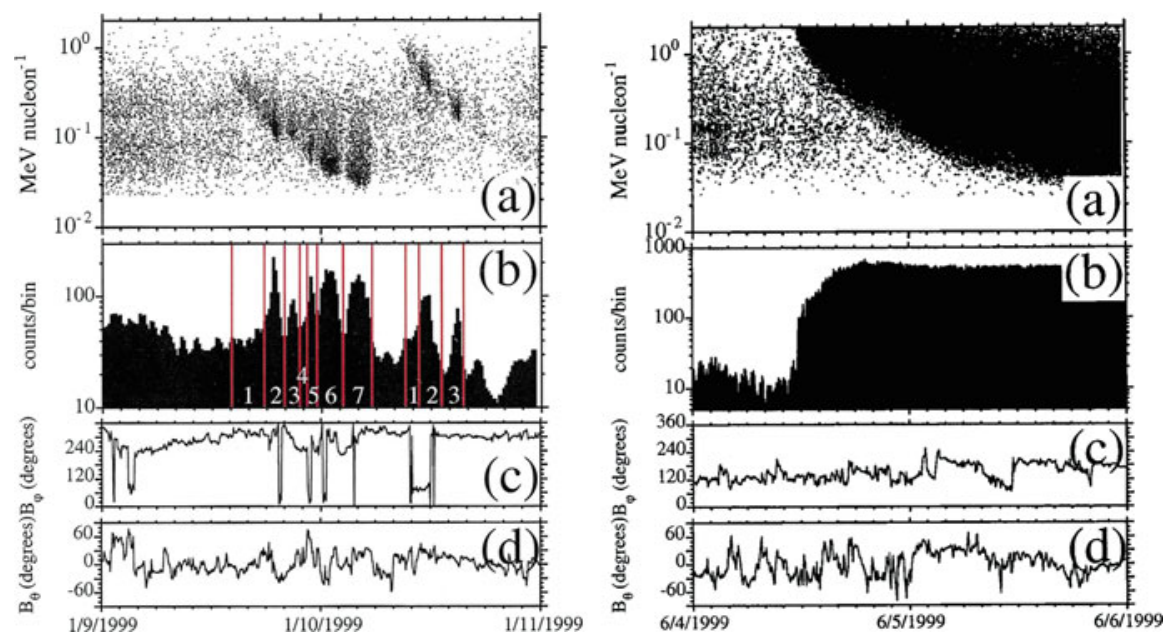

Fig. 3.8 Panels (a) show the energy vs. the arrival time of individual ions from an impulsive (left) and a gradual (right) SEP event. Panels (b) show the corresponding ion count rates while (c) and (d) show the magnetic field direction. Particles or gaps occur because some flux tubes connect to the compact source of impulsive SEPs and others do not (Mazur et al. 2000, C) AAS)

Subsequent observations (Chollet and Giacalone 2011) found the boundaries between flux tubes with and without SEPs were extremely sharp. This indicated that there was little or no cross-field transport. The mixing of magnetic flux tubes that do and do not connect to any specific location on the Sun is expected from the random walk of their footpoints prior to the particle event (see Sect. 2.3.5).

More-recent observations (Tan and Reames 2016; Tan 2017) have shown that there are occasional dropouts during gradual SEP events as well. Differences in the scattering in some magnetic flux tubes can have an especially strong affect on the intensities and angular distributions of non-relativistic electrons. These profound sudden changes in particle intensities mostly occur when differently-connected flux tubes are sampled inside a passing CME, as measured by multiple spacecraft in the SEP event of 14 December 2006 (von Rosenvinge et al. 2009).

\subsection{Abundances}

Abundances of elements and isotopes were one of the earliest indications of the two different sources of SEPs:

1. The average abundances of the elements in gradual events, relative to those in the 
photosphere, measured a FIP pattern related to the abundances in the corona and solar wind (see Sects. 1.5.2 and 2.5.1). Since they were associated with fast, wide CMEs driving shock waves, this fit well with the idea of a shock wave sampling ambient coronal abundances.

2. The strong 1000-fold enhancements of ${ }^{3} \mathrm{He} /{ }^{4} \mathrm{He}$, and the associations with streaming electrons, and with the type III radio bursts they produce, were clearly related to an impulsive source at the Sun and soon connected with narrow CMEs and solar jets (see Kahler et al. 2001; Reames et al. 2014a; Bučík et al. 2018; see also Sects. 2.5.2 and 4.7).

In the next chapters (Sects. 4.6 and 5.6) we will see that the pattern of the powerlaw dependence of abundance enhancements on $A / Q$ of the ions leads to a determination of $Q$ values and of the associated source-plasma temperature $T$. The results are:

1. Gradual events: $\sim 69 \%$ of events $0.8<T<1.6 \mathrm{MK}, 24 \%$ of events $T=2-4 \mathrm{MK}$ from re-accelerated impulsive SEP seed ions (Reames 2016).

2. Impulsive SEP events: $\mathrm{T}=2-4 \mathrm{MK}$ (Reames et al. 2014a, b).

Thus in $69 \%$ of gradual events, shocks sweep up material at ambient coronal temperatures. In $24 \%$, shocks traverse active regions and re-accelerate some residual impulsive suprathermal ions diluted by some ambient active-region plasma, especially protons. We will develop the techniques for determining source temperatures in Sects. 4.6 and 5.6. The temperatures are strong evidence for shock acceleration of large gradual SEP events. Ambient coronal temperatures of SEPs would seem to be hard to explain for those who would like to accelerate gradual SEPs in hot flares, or even to store SEPs in hot flare loops. Furthermore, GLEs show a similar distribution of source-plasma temperatures and abundances as non-GLEs. We find nothing unique about the physics of GLEs; they just happen to have harder spectra and direct a few more high-energy particles toward Earth.

\subsection{Electrons}

In a review article, Ramaty et al. (1980) studied peak intensities of $0.5-1.1 \mathrm{MeV}$ electrons vs. those of $10 \mathrm{MeV}$ protons. For sufficiently intense protons they found a correlation between the electrons and protons that they ascribed to common acceleration by a shock wave. Cliver and Ling (2007) revisited this study from the perspective of impulsive and gradual SEP events. Their interesting findings are shown in Fig. 3.9.

This clever study makes use of the fact that impulsive events are nearly all magnetically well-connected. The known impulsive events show no evidence of electron-proton intensity correlation. The shock-accelerated events span a much larger region of solar longitude and show electron-proton correlation where the 

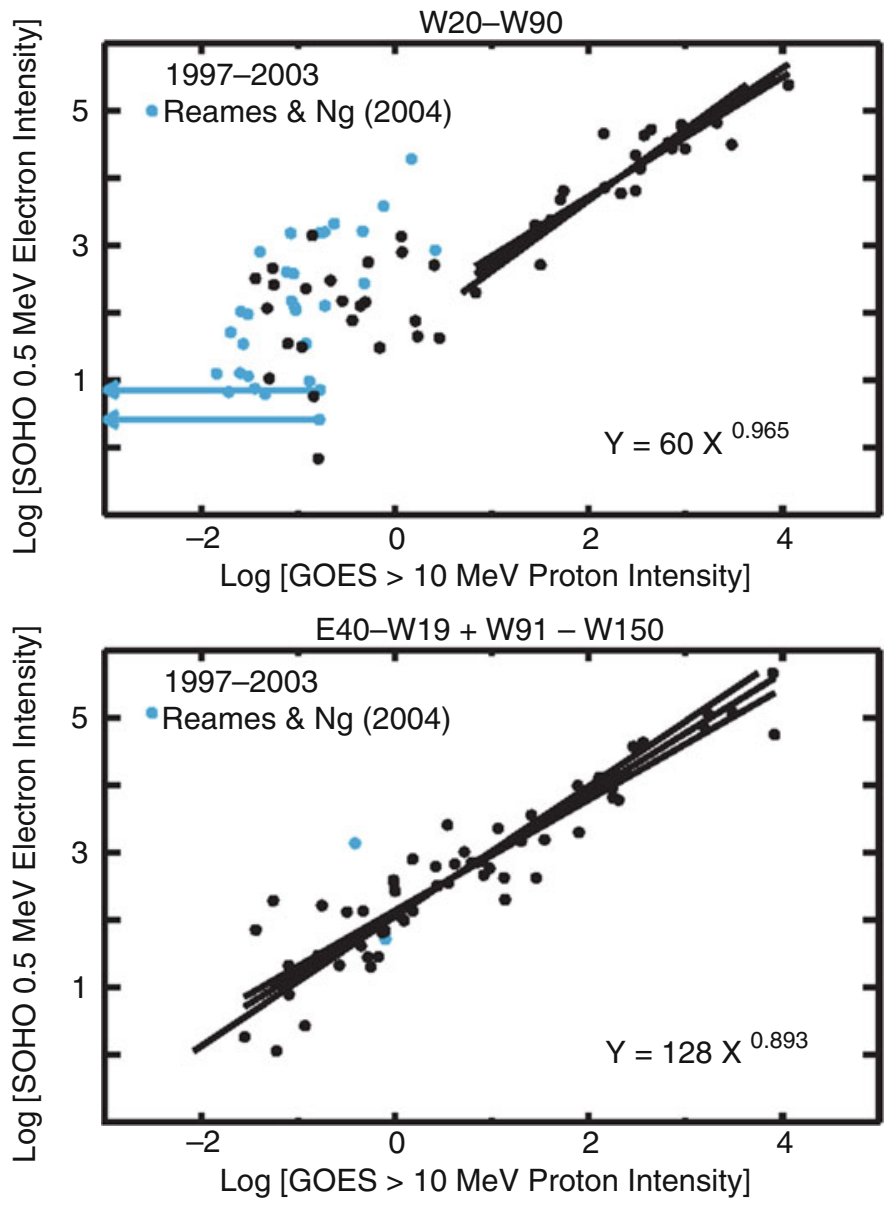

Fig. 3.9 The panels each show peak $0.5-\mathrm{MeV}$ electron intensity vs. peak $10-\mathrm{MeV}$ proton intensity. Events in the upper panel are well connected (20-90 W) while those in the lower panel are poorly connected. The events in blue are impulsive events that are ${ }^{3} \mathrm{He}$-rich with enhanced heavy elements. A proton-intense subset of well-connected events shows a strong correlation while essentially all of the poorly-connected events are correlated (Cliver and Ling 2007 (C) AAS)

impulsive events are absent, i.e. events that are poorly connected or those that have high proton intensities.

Are the $0.5-\mathrm{MeV}$ electrons accelerated at the same shock as the $10-\mathrm{MeV}$ protons? Apparently so. In general, it is difficult to know how to compare protons and electrons. Unfortunately, low-energy electrons do not resonate with Alfvén waves as low-energy protons do. Should they be compared at the same energy, rigidity, or velocity? Usually the available intervals are used that are neither; yet, despite the lack of an ideal variable for comparison, it is still usually possible to conclude that 
impulsive SEPs are electron rich, and that shocks in gradual events accelerate both protons and relativistic electrons.

Recently, an extensive list of over 1000 electron events spanning a solar cycle was prepared by Wang et al. (2012). The list includes radio, CME, X-ray, flare, ${ }^{3} \mathrm{He} /{ }^{4} \mathrm{He}$, and $10-\mathrm{MeV}$ proton data.

Electron acceleration at shock waves is a historical problem. There is even lore that shocks cannot accelerate electrons While ions can scatter back and forth against Alfvén waves at the quasi-parallel nose, where the shock is fastest, non-relativistic electrons cannot resonate with Alfvén waves and are not similarly accelerated. However, electrons can be accelerated in the $\boldsymbol{V}_{\mathbf{S}} \times \boldsymbol{B}$ electric fields on the oblique flanks of the shock.

Are type-II radio bursts only emitted from the flanks of shocks? No. They come from the nose as well. Type-II emission from the shock nose and flanks can be distinguished because the nose is farther out from the Sun, at lower $n_{\mathrm{e}}$, and therefore lower frequency; the frequencies can be correlated with the coronagraph image. Actually, type-II emission from the flanks of a shock goes by the special name: diffuse interplanetary radio emission (DIRE; e.g. Gopalswamy 2020).

Electron acceleration at the quasi-parallel nose of a shock probably occurs because real shocks are not planar but are very complex structures, varying in space and time. This complexity was even noted in Jones and Ellison (1991) and has been observed directly in interplanetary shocks with the Cluster spacecraft by Kajdič et al. (2019). The fluctuations can even involve $\theta_{\mathrm{Bn}}$ so $\boldsymbol{V}_{\mathbf{S}} \times \boldsymbol{B}$ electric fields may be available everywhere to accelerate electrons. Electrons are certainly accelerated at shocks, they produce type-II emission and they even manage to reach relativistic energies as seen in Fig. 3.9.

\subsection{Why Not Flares?}

Impulsive SEP events in space are produced by solar jets, not flares. Solar flares exist precisely because the SEPs in them are magnetically trapped. Sites of electron acceleration in magnetic reconnection occur above the newly-forming closed magnetic loops at heights of $>20 \mathrm{Mm}$ and at densities of a few times $10^{9} \mathrm{~cm}^{-3}$ (Krucker et al. 2010; Krucker and Battaglia 2014). Ion acceleration is likely to occur at the same location (e.g. Drake et al. 2009). These SEPs eventually scatter into the dense regions of the loop footpoints where they dump all their energy, causing heating and expansion of hot, bright plasma back up into the loops. Observations of $\gamma$-ray lines have shown that the energetic ions in flares are ${ }^{3} \mathrm{He}$-rich (Mandzhavidze et al. 1999; Murphy et al. 2016) and Fe-rich (Murphy et al. 1991), just like the impulsive SEP events we see in space. However, these $\gamma$-rays arise from nuclear reactions that also produce secondary ions of ${ }^{2} \mathrm{H},{ }^{3} \mathrm{H}$, and isotopes of $\mathrm{Li}, \mathrm{Be}$, and $\mathrm{B}$. These reaction products are not seen in space. Serlemitsos and Balasubrahmanyan (1975) found ${ }^{3} \mathrm{He} /{ }^{4} \mathrm{He}=1.52 \pm 0.10$, compared with $(4.08 \pm 0.25) \times 10^{-4}$ in the solar wind 
(Gloeckler and Geiss 1998), but they also found ${ }^{3} \mathrm{He} /{ }^{2} \mathrm{H}>300$. Limits on Be/O or $\mathrm{B} / \mathrm{O}$ in large SEP events are $<2 \times 10^{-4}$ (e.g. McGuire et al. 1979; Cook et al. 1984). Neutral $\gamma$-rays and neutrons (Chupp et al. 1982; Evenson et al. 1983, 1990) from nuclear reactions escape, but SEPs that actually caused the flare never escape the closed magnetic loops. One cannot exclude a stray leak from a flare, but that must be swamped by SEPs from the shock source.

In Carrington's (1860) first observation of a flare, he was "surprised . . . at finding myself unable to recognize any change whatever as having taken place." The fields maintain their shape. Modern instruments allow measurement of the reconnection magnetic flux and a recent data base contains reconnection flux for 3137 solar-flare ribbon events (Kazachenko et al. 2017). Large reconnection events that lacked fast shock waves produce beautiful flares, but no SEPs (Kahler et al. 2017). Flares require containment by closed magnetic fields and disrupt the general field topology very little; the field energy transmitted to particles accelerated in flares is dissipated as heat in the footpoints and loops and we do not see these energetic particles in space.

Historically, it has been the neutron monitors that have been used most often to try to associate a flare source with GLEs. These are the biggest events, where everything happens, and are the most prone to the errors of causal association that result from "big flare syndrome" (Kahler 1982). Worse yet, neutron monitors do not measure particle abundances, their energy measurements are limited, and anisotropic intensities of streaming $\mathrm{GeV}$ protons are often modulated by variations in the direction of the interplanetary magnetic field relative to the asymptotic look direction of a site. At best, neutron monitors mainly measure timing, and many physical processes, including shock acceleration, can be fast.

We prefer to study SEPs measured in the smallest events, to identify sources. Jets (Sect. 4.7) without fast shocks give us impulsive SEP events, and disappearingfilament events (Sect. 2.7) drive fast shocks with no flares or jets, yet they give us gradual SEP events that depend upon the shock properties. These smaller, simpler single-source events better define SEP origins.

Flares are bright because they are hot, reaching temperatures if 10-40 MK. When we measure the source plasma temperatures of SEPs (see Sects. 2.6, 4.6 and 5.6), we find temperatures of 1-4 MK, i.e. ambient temperatures of coronal plasma. At 10-40 $\mathrm{MK}$, even the elements $\mathrm{Ne}, \mathrm{Mg}$, and $\mathrm{Si}$ become fully ionized so they all have $\mathrm{A} /$ $Q=2$, just like $\mathrm{He}, \mathrm{C}, \mathrm{N}$, and $\mathrm{O}$. This hot plasma would not support the observed enhancements seen in $\mathrm{Ne} / \mathrm{O}$ or even $\mathrm{Si} / \mathrm{O}$ (Chap. 4). SEPs from hot flare plasma are not seen. The observed properties of SEPs in space are not compatible with such hot flare plasma and closed-field regions do not just open to release them. The SEP sources we once attributed to specific flares most likely come from nearby jets where the SEPs escape rapidly with minimal heating.

To the extent that SEPs in flares are similar to those in jets, flare observations, especially $\gamma$-ray lines, could be a benefit in understanding the physics of impulsive 
acceleration mechanisms. It would be helpful to have more-extensive measurements of $\gamma$-ray lines (Sect. 4.9).

\subsection{SEPs as Probes}

As SEPs stream out along the interplanetary field they can map its structure. This was shown early by the radio mapping of the electron population in type III radio bursts. Knowing the direction to the center of the radio signal and its distance from the Sun determined by the frequency and models of the electron density vs. radius, the electrons could be followed, as seen in Fig. 3.10. Occasionally, trajectories of this kind can be made using triangulation from two or more spacecraft (Reiner et al. 1998; see also Li et al. 2016). While such electron trajectories generally follow the Parker spiral, it is important to realize that field lines are often distorted by variations in the solar-wind speed and by the passage of CMEs.

Fig. 3.10 The trajectory of the electron population in the type III radio burst accompanying a ${ }^{3} \mathrm{He}$-rich SEP event is shown in three dimensions (a) and as a projection on the ecliptic (b) (Reames and Stone 1986 (C) AAS)

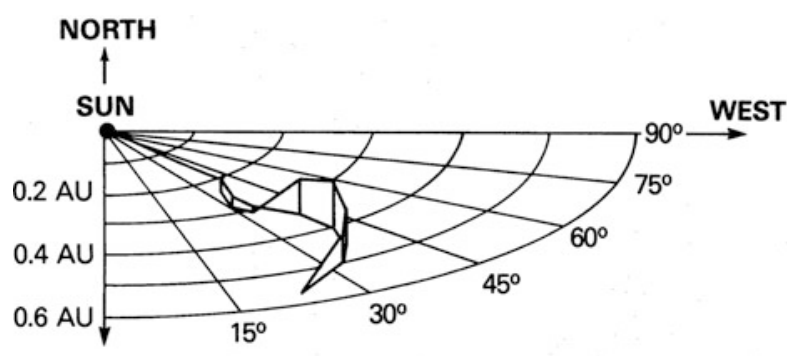

a

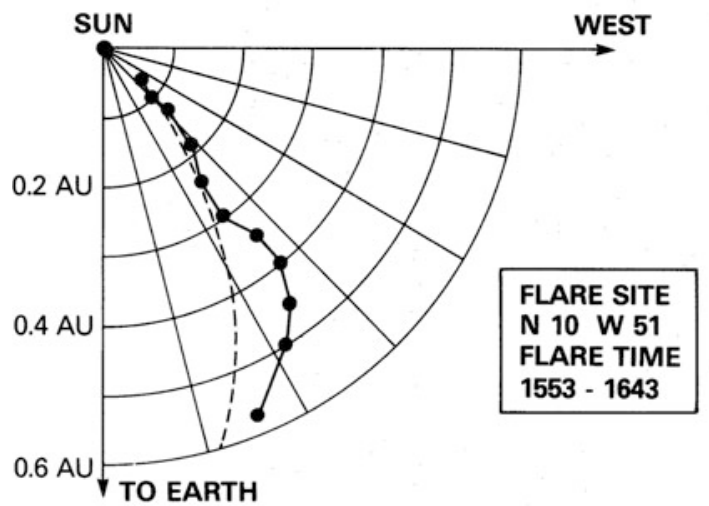




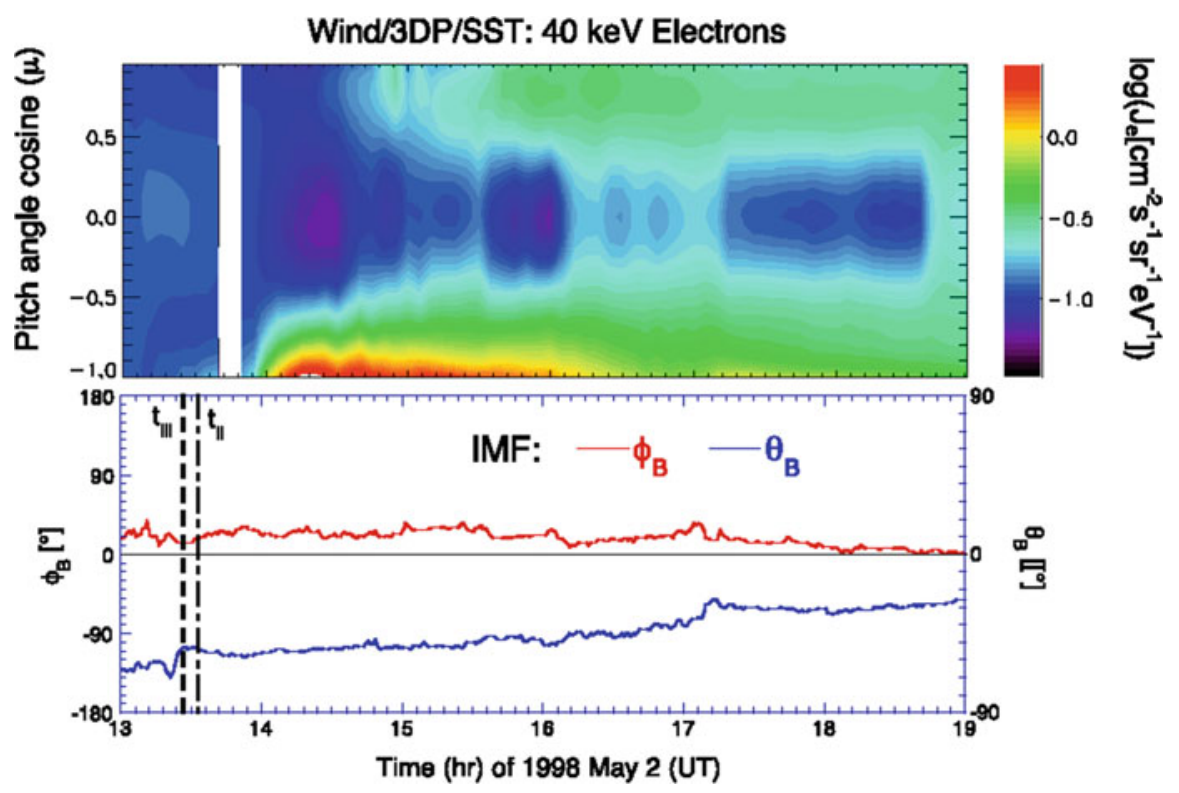

Fig. 3.11 The pitch-angle spectrogram of $40 \mathrm{keV}$ electrons from the 2 May 1998 SEP event is shown in the upper panel. Electrons first appear from the Sun at $\mu \approx-1$ near 1400 UT and reflected electrons appear at $\mu \approx+1$ around 1500 UT. The lower panels show the field direction (Tan et al. 2013, (C) AAS)

Low-energy $(<100 \mathrm{keV})$ electrons are often seen passing Earth outbound then returning sunward from a magnetic reflection site beyond Earth (Kahler and Reames 1991; Tan et al. 2012, 2013) as shown in the example in Fig. 3.11. These electrons have highly scatter-free transport (Tan et al. 2011) and are thus excellent probes of the magnetic topology.

Figure 3.12 uses separate incident and reflected electron onset times to determine the release times and pathlengths for each, and also shows He ions, with no reflected beam (Tan et al. 2012). Pathlengths for the incident ions and electrons are quite similar and near the length of the Parker spiral. Electrons depart about 13 min earlier. Reflected electrons travel $\approx 1$ AU farther in this event.

However, electrons are not the only particles affected by their journey, Reames and $\mathrm{Ng}$ (2002) found that in some SEP events Fe/O was higher for sunward bound ions than for those that were outward bound. Since Fe scatters less then O, the Fe more rapidly passed Earth to be reflected sunward than O, so the returning particles were more Fe-rich, i.e. they had simply traveled farther from the source.

There should be no surprise that the magnetic fields depart from the simple Parker spiral and become quite complex. Not only is there the random walk of field lines 

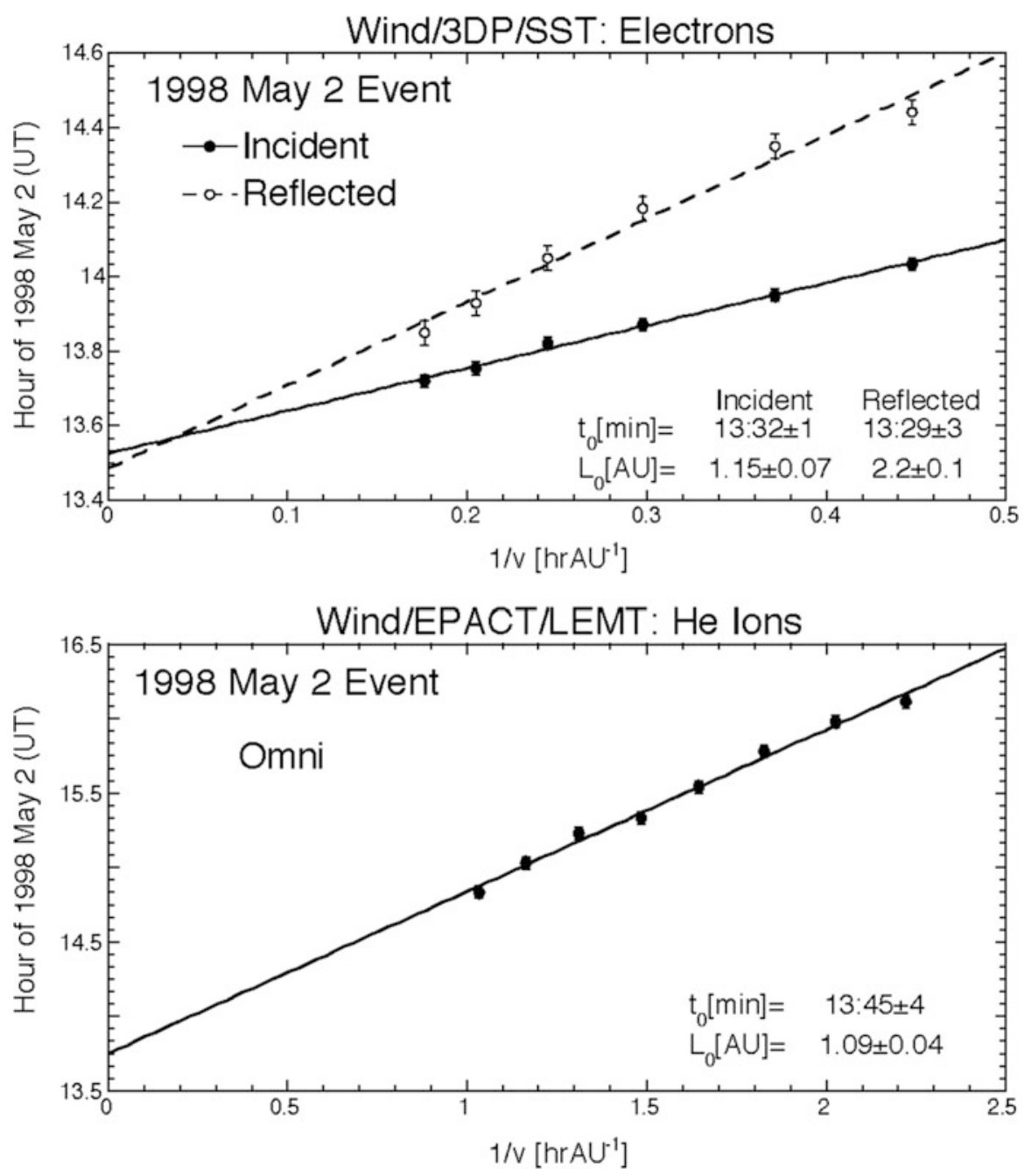

Fig. 3.12 The upper panel shows the onset times of both incident and reflected electrons vs. $v^{-1}$ with the fitted release time and pathlength for each. The lower panel shows omni-directional data for He (Tan et al. 2012, (C) AAS). The He SEPs are actually highly collimated for a day after onset; they show no reflection at the same time as the reflected electrons

discussed in Sect. 2.3.5, but there is a constant progression of CMEs that disturb the field as suggested by Fig. 3.13.

The Sun can eject 2.5 CMEs day ${ }^{-1}$ at solar maximum (Webb and Howard 1994). If each CME occupies one steradian and its typical speed is $\sim 400 \mathrm{~km} \mathrm{~s}^{-1}$, CMEs will be randomly spaced at radial distances of typically $\sim 1 \mathrm{AU}$ apart, one after the other, out into the heliosphere in any direction. While most of them would be too slow to 


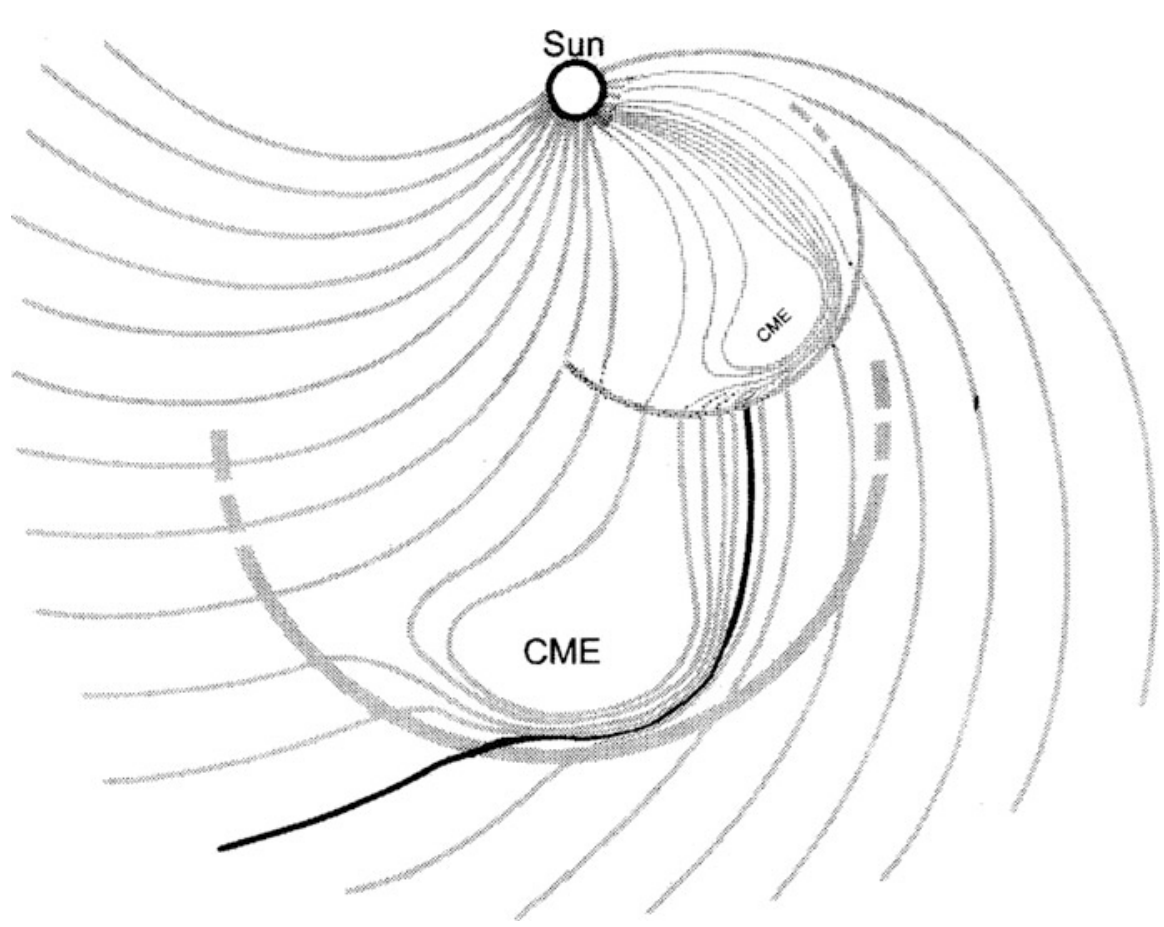

Fig. 3.13 The Sun can emit a progression of CMEs as suggested here. Particles accelerated at a shock preceding the smaller, newer CME can easily be reflected by converging field lines of the outer CME or by turbulence at the shock that precedes it

generate shocks, they would have low $\beta_{\mathrm{P}}$ and would carry magnetic flux ropes that contribute to magnetic distortions capable of reflecting particles.

Bidirectional flows of protons or electrons are commonly seen within CMEs near 1 AU (e.g. Kahler and Reames 1991; Richardson and Reames 1993). These flows are often used as probes to study the topology of the magnetic fields, and even to identify CMEs. Their common occurrence also shows that pitch-angle scattering is minimal within CMEs.

\section{References}

Bučík, R., Innes, D.E., Mason, G.M., Wiedenbeck, M.E., Gómez-Herrero, R., Nitta, N.: ${ }^{3}$ He-rich solar energetic particles in helical jets on the sun. Astrophys. J. 852, 76 (2018). https://doi.org/ 10.3847/1538-4357/aa9d8f

Carrington, R.C.: Description of a singular appearance seen in the Sun on September, 1859. Mon. Not. Roy. Astron. Soc. 20, 13 (1860). https://doi.org/10.1093/mnras/20.1.13

Chollet, E.E., Giacalone, J.: Evidence of confinement of solar-energetic particles to interplanetary magnetic field lines. Astrophys. J. 728, 64 (2011). https://doi.org/10.1088/0004-637X/728/1/64 
Chupp, E.L., Forrest, D.J., Ryan, J.M., Heslin, J., Reppin, C., Pinkau, K., Kanbach, G., Rieger, E., Share, G.H.: A direct observation of solar neutrons following the 0118 UT flare on 1980 June 21. Astrophys. J. Lett. 263, L95 (1982). https://doi.org/10.1086/183931

Cliver, E.W., Ling, A.G.: Electrons and protons in solar energetic particle events. Astrophys. J. 658, 1349 (2007). https://doi.org/10.1086/511737

Cliver, E.W., Kahler, S.W., Reames, D.V.: Coronal shocks and solar energetic proton events. Astrophys. J. 605, 902 (2004). https://doi.org/10.1086/382651

Cook, W.R., Stone, E.C., Vogt, R.E.: Elemental composition of solar energetic particles. Astrophys. J. 279, 827 (1984). https://doi.org/10.1086/161953

Drake, J.F., Cassak, P.A., Shay, M.A., Swisdak, M., Quataert, E.: A magnetic reconnection mechanism for ion acceleration and abundance enhancements in impulsive flares. Astrophys. J. Lett. 700, L16 (2009). https://doi.org/10.1088/0004-637X/700/1/L16

Ellison, D., Ramaty, R.: Shock acceleration of electrons and ions in solar flares. Astrophys. J. 298, 400 (1985). https://doi.org/10.1086/163623

Evenson, P., Meyer, P., Pyle, K.R.: Protons from the decay of solar flare neutrons. Astrophys. J. 274, 875 (1983). https://doi.org/10.1086/161500

Evenson, P., Kroeger, R., Meyer, P., Reames, D.: Solar neutron decay proton observations in cycle 21. Astrophys. J. Suppl. 73, 273 (1990). https://doi.org/10.1086/191462

Giacalone, J., Jokipii, J.R., Mazur, J.E.: Small-scale gradients and large-scale diffusion of charged particles in the heliospheric magnetic field. Astrophys. J. Lett. 532, L78 (2000). https://doi.org/ $10.1086 / 312564$

Gloeckler, G., Geiss, J.: Measurement of the abundance of Helium-3 in the Sun and in the local interstellar cloud with SWICS on ULYSSES. Space Sci. Rev. 84, 275 (1998)

Gopalswamy, N.: Interplanetary radio emission: a summary of recent result, eprint (2020) arXiv:2008.09222

Gopalswamy, N., Xie, H., Yashiro, S., Akiyama, S., Mäkelä, P., Usoskin, I.G.: Properties of Ground level enhancement events and the associated solar eruptions during solar cycle 23. Space Sci. Rev. 171, 23 (2012). https://doi.org/10.1007/s11214-012-9890-4

Gopalswamy, N., Xie, H., Akiyama, S., Yashiro, S., Usoskin, I.G., Davila, J.M.: The first ground level enhancement event of Solar Cycle 24: direct observation of shock formation and particle release heights. Astrophys. J. Lett. 765, L30 (2013). https://doi.org/10.1088/2041-8205/765/2/ L30

Jones, F.C., Ellison, D.C.: The plasma physics of shock acceleration. Space Sci. Rev. 58, 259 (1991). https://doi.org/10.1007/BF01206003

Kahler, S.W.: The role of the big flare syndrome in correlations of solar energetic proton fluxes and associated microwave burst parameters. J. Geophys. Res. 87, 3439 (1982). https://doi.org/10. 1029/JA087iA05p03439

Kahler, S.W.: Injection profiles of solar energetic particles as functions of coronal mass ejection heights. Astrophys. J. 428, 837 (1994). https://doi.org/10.1086/174292

Kahler, S.W.: The correlation between solar energetic particle peak intensities and speeds of coronal mass ejections: Effects of ambient particle intensities and energy spectra. J. Geophys. Res. 106, 20947 (2001). https://doi.org/10.1029/2000JA002231

Kahler, S.W., Reames, D.V.: Probing the magnetic topologies of magnetic clouds by means of solar energetic particles. J. Geophys. Res. 96, 9419 (1991). https://doi.org/10.1029/91JA00659

Kahler, S.W., Reames, D.V., Sheeley Jr., N.R.: Coronal mass ejections associated with impulsive solar energetic particle events. Astrophys. J. 562, 558 (2001). https://doi.org/10.1086/323847

Kahler, S.W., Kazachenko, M., Lynch, B.J., Welsch, B.T.: Flare magnetic reconnection fluxes as possible signatures of flare contributions to gradual SEP events. J. Phys. Conf. Series. 900, 012011 (2017). https://doi.org/10.1088/1742-6596/900/1/012011

Kajdič, P., Preisser, L., Blanco-Cano, X., Burgess, D., Trotta, D.: First observations of irregular surface of interplanetary shocks at ion scales by Cluster. Astrophys. J. Lett. 874, L13 (2019). https://doi.org/10.3847/2041-8213/ab0e84 
Kazachenko, M.D., Lynch, B.J., Welsch, B.T., Sun, X.: A database of flare ribbon properties from solar dynamics observatory I: Reconnection flux. Astrophys. J. 845, 49 (2017). https://doi.org/ 10.3847/1538-4357/aa7ed6. arXiv: 1704.05097

Kouloumvakos, A., Rouillard, A., Wu, Y., Vainio, R., Vourlidas, A., Plotnikov, I., Afanasiev, A., Önel, H.: Connecting the properties of coronal shock waves with those of solar energetic particles. Astrophys. J. 876, 80 (2019). https://doi.org/10.3847/1538-4357/ab15d7

Krucker, S., Battaglia, M.: Particle densities within the acceleration region of a solar flare. Astrophys. J. 780, 107 (2014). https://doi.org/10.1088/0004-637X/780/1/107

Krucker, S., Hudson, H.S., Glesener, L., White, S.M., Masuda, S., Wuelser, J.P., Lin, R.P.: Measurements of the coronal acceleration region of a solar flare. Astrophys. J. 714, 1108 (2010). https://doi.org/10.1088/0004-637X/714/2/1108

Lee, M.A.: Coupled hydromagnetic wave excitation and ion acceleration at an evolving coronal/ interplanetary shock. Astrophys. J. Suppl. 158, 38 (2005). https://doi.org/10.1086/428753

Li, B., Cairns, I.H., Gosling, J.T., Steward, G., Francis, M., Neudegg, D., Schulte in den Bäumen, H., Player, P.R., Milne, A.R.: Mapping magnetic field lines between the Sun and Earth. J. Geophys. Res. 121, 925 (2016). doi: https://doi.org/10.1002/2015JA021853

Lovell, J.L., Duldig, M.L., Humble, J.E.: An extended analysis of the September 1989 cosmic ray ground level enhancement. J. Geophys. Res. 103, 23733 (1998). https://doi.org/10.1029/98JA02100

Mandzhavidze, N., Ramaty, R., Kozlovsky, B.: Determination of the abundances of subcoronal ${ }^{4} \mathrm{He}$ and of solar flare-accelerated ${ }^{3} \mathrm{He}$ and ${ }^{4} \mathrm{He}$ from gamma-ray spectroscopy. Astrophys. J. 518, 918 (1999). https://doi.org/10.1086/307321

Mann, G., Klassen, A., Aurass, H., Classen, H.-T.: Formation and development of shock waves in the solar corona and the near-Sun interplanetary space. Astron. Astrophys. 400, 329 (2003). https://doi.org/10.1051/0004-6361:20021593

Mazur, J.E., Mason, G.M., Dwyer, J.R., Giacalone, J., Jokipii, J.R., Stone, E.C.: Interplanetary magnetic field line mixing deduced from impulsive solar flare particles. Astrophys. J. Lett. 532, L79 (2000). https://doi.org/10.1086/312561

McGuire, R.E., von Rosenvinge, T.T., McDonald, F.B.: A survey of solar cosmic ray composition. Proceedings of the 16th International Cosmic Ray Conference, Tokyo. 5, 61 (1979)

Mewaldt, R.A., Looper, M.D., Cohen, C.M.S., Haggerty, D.K., Labrador, A.W., Leske, R.A., Mason, G.M., Mazur, J.E., von Rosenvinge, T.T.: Energy spectra, composition, other properties of ground-level events during solar cycle 23. Space Sci. Rev. 171, 97 (2012). https://doi.org/10. 1007/s11214-012-9884-2

Murphy, R.J., Ramaty, R., Kozlovsky, B., Reames, D.V.: Solar abundances from gamma-ray spectroscopy: Comparisons with energetic particle, photospheric, and coronal abundances. Astrophys. J. 371, 793 (1991). https://doi.org/10.1086/169944

Murphy, R.J., Kozlovsky, B., Share, G.H.: Evidence for enhanced ${ }^{3} \mathrm{He}$ in flare-accelerated particles based on new calculations of the gamma-ray line spectrum. Astrophys. J. 833, 196 (2016). https://doi.org/10.3847/1538-4357/833/2/196

Ramaty, R., Paizis, C., Colgate, S.A., Dulk, G.A., Hoyng, P., Knight, J.W., Lin, R.P., Melrose, D. B., Orrall, F., Shapiro, P.R.: Energetic particles in solar flares. In: Solar flares: a monograph from Skylab Solar Workshop II (A80-37026 15-92). Colorado Associated University Press, Boulder, Colo, p 117 (1980)

Reames, D.V.: Solar release times of energetic particles in ground-level events. Astrophys. J. 693, 812 (2009a). https://doi.org/10.1088/0004-637X/693/1/812

Reames, D.V.: Solar energetic-particle release times in historic ground-level events. Astrophys. J. 706, 844 (2009b). https://doi.org/10.1088/0004-637X/706/1/844

Reames, D.V.: Temperature of the source plasma in gradual solar energetic particle events. Sol. Phys. 291, 911 (2016). https://doi.org/10.1007/s11207-016-0854-9. arXiv: 1509.08948

Reames, D.V., Lal, N.: A multi-spacecraft view of solar-energetic-particle onsets in the 1977 November 22 event. Astrophys. J. 723, 550 (2010). https://doi.org/10.1088/0004-637X/723/1/550

Reames, D.V., Ng, C.K.: Angular distributions of Fe/O from wind: new insight into solar energetic particle transport, Astrophys. J. Lett. 575, L37 (2002). https://doi.org/10.1086/344146 
Reames, D.V., Ng, C.K.: Heavy-element abundances in solar energetic particle events. Astrophys. J. 610, 510 (2004). https://doi.org/10.1086/421518

Reames, D.V., Stone, R.G.: The identification of solar He-3-rich events and the study of particle acceleration at the sun. Astrophys. J. 308, 902 (1986). https://doi.org/10.1086/164560

Reames, D.V., Cliver, E.W., Kahler, S.W.: Abundance enhancements in impulsive solar energeticparticle events with associated coronal mass ejections. Sol. Phys. 289, 3817 (2014a). https://doi. org/10.1007/s11207-014-0547-1

Reames, D.V., Cliver, E.W., Kahler, S.W.: Variations in abundance enhancements in impulsive solar energetic-particle events and related CMEs and flares. Sol. Phys. 289, 4675 (2014b). https://doi.org/10.1007/s11207-014-0589-4

Reiner, M.J., Fainberg, J., Kaiser, M.L., Stone, R.G.: Type III radio source located by Ulysses/Wind triangulation. J. Geophys. Res. 103, 1923 (1998). https://doi.org/10.1029/97JA02646

Richardson, I.G., Reames, D.V.: Bidirectional $\sim 1 \mathrm{MeV} \mathrm{amu}^{-1}$ ion intervals in 1973--1991 observed by the Goddard Space Flight Center instruments on IMP 8 and ISEE 3/ICE. Astrophys. J. Suppl. $\mathbf{8 5}, 411$ (1993). https://doi.org/10.1086/191769

Rouillard, A.C., Odstrčil, D., Sheeley Jr., N.R., Tylka, A.J., Vourlidas, A., Mason, G., Wu, C.-C., Savani, N.P., Wood, B.E., Ng, C.K., et al.: Interpreting the properties of solar energetic particle events by using combined imaging and modeling of interplanetary shocks. Astrophys. J. 735, 7 (2011). https://doi.org/10.1088/0004-637X/735/1/7

Rouillard, A., Sheeley Jr., N.R., Tylka, A., Vourlidas, A., Ng, C.K., Rakowski, C., Cohen, C.M.S., Mewaldt, R.A., Mason, G.M., Reames, D., et al.: The longitudinal properties of a solar energetic particle event investigated using modern solar imaging. Astrophys. J. 752, 44 (2012). https:// doi.org/10.1088/0004-637X/752/1/44

Rouillard, A.P., Plotnikov, I., Pinto, R.F., Tirole, M., Lavarra, M., Zucca, P., Vainio, R., Tylka, A. J., Vourlidas, A., De Rosa, M.L., Linker, J., Warmuth, A., Mann, G., Cohen, C.M.S., Mewaldt, R.A.: Deriving the properties of coronal pressure fronts in 3D: application to the 2012 May 17 ground level enhancement. Astrophys. J. 833, 45 (2016). https://doi.org/10.3847/1538-4357/ $833 / 1 / 45$

Serlemitsos, A.T., Balasubrahmanyan, V.K.: Solar particle events with anomalously large relative abundance of ${ }^{3} \mathrm{He}$. Astrophys. J. 198, 195 (1975). https://doi.org/10.1086/153592

Tan, L.C.: Electron-ion intensity dropouts in gradual solar energetic particle events during solar cycle 23. Astrophys. J. 846, 18 (2017). https://doi.org/10.3847/1538-4357/aa81d1

Tan, L.C., Reames, D.V.: Dropout of directional electron intensities in large solar energetic particle events. Astrophys. J. 816, 93 (2016). https://doi.org/10.3847/0004-637X/816/2/93

Tan, L.C., Reames, D.V., Ng, C.K., Shao, X., Wang, L.: What causes scatter-free transport of non-relativistic solar electrons? Astrophys. J. 728, 133 (2011). https://doi.org/10.1088/0004$637 \mathrm{X} / 728 / 2 / 133$

Tan, L.C., Malandraki, O.E., Reames, D.V., Ng, C.K., Wang, L., Dorrian, G.: Use of incident and reflected solar particle beams to trace the topology of magnetic clouds. Astrophys. J. 750, 146 (2012). https://doi.org/10.1088/0004-637X/750/2/146

Tan, L.C., Malandraki, O.E., Reames, D.V., Ng, C.K., Wang, L., Patsou, I., Papaioannou, A.: Comparison between path lengths traveled by solar electrons and ions in ground-level enhancement events. Astrophys. J. 768, 68 (2013). https://doi.org/10.1088/0004-637X/768/1/68

Thakur, N., Gopalswamy, N., Mäkelä, P., Akiyama, S., Yashiro, S., Xie, H.: Two exceptions in the large SEP events of Solar Cycles 23 and 24. Solar. Phys. 231, 519 (2016). https://doi.org/10. 1007/s11207-015-0830-9

Tylka, A.J., Dietrich, W.F.: A new and comprehensive analysis of proton spectra in ground-level enhanced (GLE) solar particle events. In Proceedings of the 31st International Cosmic Ray Conference Lódz (2009). http://icrc2009.uni.lodz.pl/proc/pdf/icrc0273.pdf

Tylka, A.J., Cohen, C.M.S., Dietrich, W.F., Krucker, S., McGuire, R.E., Mewaldt, R.A., Ng, C.K., Reames, D.V, Share, G.H.: Onsets and release times in solar particle events. In: Proceedings of the 28th Internation Cosmic Ray Conference, 3305 (2003) 
von Rosenvinge, T.T., Richardson, I.G., Reames, D.V., Cohen, C.M.S., Cummings, A.C., Leske, R.A., Mewaldt, R.A., Stone, E.C., Wiedenbeck, M.E.: The solar energetic particle event of 14 December 2006. Sol. Phys. 256, 443 (2009). https://doi.org/10.1007/s11207-009-9353-6

Wang, L., Lin, R.P., Krucker, S., Mason, G.M.: A statistical study of solar electron events over one solar cycle. Astrophys. J. 759, 69 (2012). https://doi.org/10.1088/0004-637X/759/1/69

Webb, D.F., Howard, R.A.: The solar cycle variation of coronal mass ejections and the solar wind mass flux. J. Geophys. Res. 99, 4201 (1994). https://doi.org/10.1029/93JA02742

Open Access This chapter is licensed under the terms of the Creative Commons Attribution 4.0 International License (http://creativecommons.org/licenses/by/4.0/), which permits use, sharing, adaptation, distribution and reproduction in any medium or format, as long as you give appropriate credit to the original author(s) and the source, provide a link to the Creative Commons license and indicate if changes were made.

The images or other third party material in this chapter are included in the chapter's Creative Commons license, unless indicated otherwise in a credit line to the material. If material is not included in the chapter's Creative Commons license and your intended use is not permitted by statutory regulation or exceeds the permitted use, you will need to obtain permission directly from the copyright holder.

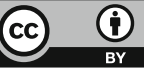

